\title{
Gaussian noise removal by color morphology and polar color models
}

\author{
Francisco Ortiz \\ Image Technological Group \\ Dept. Physics, Systems Engineering and Signal Theory \\ University of Alicante \\ Ap. Correos 99, 03080 Alicante, Spain \\ fortiz@ua.es
}

\begin{abstract}
This paper deals with the use of morphological filters by reconstruction of the mathematical morphology for Gaussian noise removal in color images. These new vector connected have the property of suppressing details preserving the contours of the objects. For the extension of the mathematical morphology to color images we chose a new polar color space, the 11 -norme. This color model guarantees the formation of the complete lattice necessary in mathematical morphology avoiding the drawbacks of others polar spaces. Finally, after having defined the vectorial geodesic operators, the opening and closing by reconstruction are then employed for the Gaussian noise elimination.
\end{abstract}

\section{Introduction}

In image processing, one of the most important tasks is the improving of the visual presentation or the reduction of noise. The main problem in noise removal is to get a clean image, which is without noise, but keeping all attributes of the original image as could be the shape, size, color, disposition and edges, among others. Some methods have been proposed so far for the elimination of noise. In [1], a directional vectorial filter is defined, which minimises the angle between vectors to eliminate the noisy pixel. In [2], the so-called vectorial median filters are an extension of the scalar median filter. In such a case, the ordering of the vectors is done with a Euclidean distance value. In [3], a combination of morphological alternate filters is used with RGB to reduce the impulsive noise.

In this paper, we show the utility of using the vectorial geodesic reconstruction in combination with a mean filter for eliminating Gaussian noise from the color images. Section 2 presents the color space chosen for mathematical processing, the l1-norme. In Section 3, we extend the geodesic operations to color images. In Section 4, we apply the vector-connected filters for eliminating the Gaussian noise in color images. Finally, our conclusions are outlined in the final section. 


\section{Color mathematical morphology}

Mathematical morphology is a non-linear image processing approach which is based on the application of lattice theory to spatial structures [4]. The definition of morphological operators needs a totally ordered complete lattice structure [5]. The application of mathematical morphology to color images is difficult, due to the vectorial nature of the color data (RGB, CMY, HLS, YIQ...). Many works have been carried out on the application of mathematical morphology to color images $[6,7,8,9,10]$. The most commonly adopted approach is based on the use of a lexicographical order which imposes a total order on the vectors. This way, we avoid the false colors in an individual filtering of signals. Let $\mathbf{x}=\left(\mathrm{x}_{1}, \mathrm{x}_{2}, \ldots, \mathrm{x}_{\mathrm{n}}\right)$ and $\mathbf{y}=\left(\mathrm{y}_{1}, \mathrm{y}_{2}, \ldots, \mathrm{y}_{\mathrm{n}}\right)$ be two arbitrary vectors $\left(\mathbf{x}, \mathbf{y} \in Z^{\mathrm{n}}\right)$. An example of lexicographical order $o_{l e x}$, will be:

$$
\mathbf{x}<\mathbf{y} \text { if }\left\{\begin{array}{c}
x_{1}<y_{1} \text { or } \\
x_{1}<y_{1} \text { and } x_{2}<y_{2} \text { or } \\
x_{1}<y_{1} \text { and } x_{2}<y_{2} \ldots \text { and... } x_{n}<y_{n}
\end{array}\right.
$$

On the other hand, it is important to define the color space in which operations are to be made. The intuitive systems (HSI, HLS, HSV,...) are used in color vision because they represent the information in a similar way to the human brain. The preference or disposition of the components of the HSI in the lexicographical ordering depends on the application and the properties of the image. Ordering with luminance (intensity) in the first position is the best way of preserving the contours of the objects in the image (lattice influenced by intensity). In situations in which the objects of interest are highly colored or in which only objects of a specific color are of interest, the operations with hue in the first position are the best (lattice influenced by hue).

\subsection{The new color space for processing: L1-norme}

There are a great number of variations for the transformation of RGB to polar color spaces (HSI, HLS HSV...). Some transformations cause that these spaces present incoherences that prevent the use of these color representations in some image

processing. These chromatic models are inadequate for the quantitative treatment of the images. For example, some instability arises in saturation of these spaces for small variations of RGB values. In addition, the saturation of the primary colors is not visually agreeable. An advisable representation must be based in distances or norms for the vectors and to provide independence between the chromatic and achromatic signals.

In order to avoid the inconveniences of HSI, HLS or HSV color models, we use, in our investigation, the new Serra's L1-norme [11]. Figure 1 shows the MS diagram from Serra's L1-norme as a positive projection of all the corners of the RGB cube in a normalization of the achromatic line to the $m$ called signal. 
This new chromatic representation has been very useful in brightness elimination of color images $[12,13]$. The intensity (achromatic signal) $m$ and saturation signal $s$ in the 11 -norme are calculated from $r, g$ and $b$ values of RGB, where the $m$ signal calculated is a normalization $(0 \leq m \leq 255)$ of the achromatic axes of the RGB cube, and the $s$ values are visually more agreeable with respect to the saturation of HLS or HSV spaces:

$$
\left\{\begin{array}{l}
m=\frac{1}{3}(r+g+b) \\
s= \begin{cases}\frac{1}{2}(2 r-g-b)=\frac{3}{2}(r-m) & \text { if }(b+r) \geq 2 g \\
\frac{1}{2}(r+g-2 b)=\frac{3}{2}(m-b) & \text { if }(b+r)<2 g\end{cases}
\end{array}\right.
$$

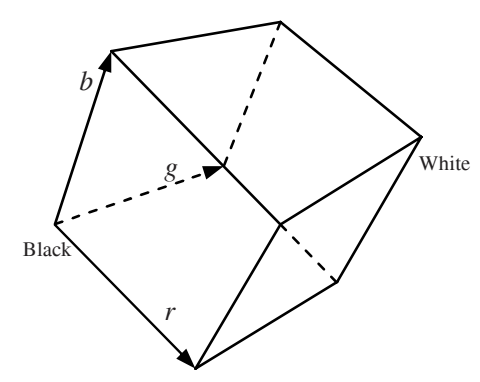

(a)

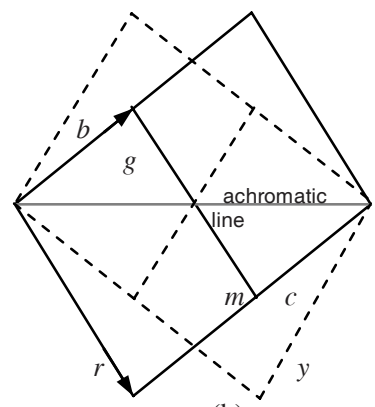

(b)

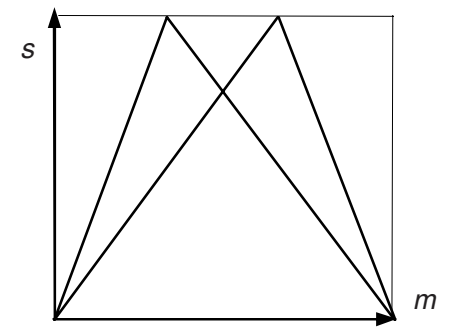

(c)

Fig. 1. RGB cube and its transformation in MS diagram. (a) 3D projection. (b) $2 \mathrm{D}$ opposite projections. (c) Shape and limits of MS diagram.

\section{Vector connected filters}

Morphological filters by reconstruction have the property of suppressing details, preserving the contours of the remaining objects $[14,15]$. The use of these filters in color images requires an ordering relationship among the pixels of the image. For the vectorial morphological processing the lexicographical ordering previously 
defined $o_{\text {lex }}$ will be used. As such, the infimum $\left(\wedge_{v}\right)$ and supremum $\left(\vee_{v}\right)$ will be vectorial operators, and they will select pixels according to their order $o_{l e x}$ in the $l 1$ norme.

Once the orders have been defined, the morphological operators of reconstruction for color images can be generated and applied. A elementary geodesic operation is the geodesic dilation. Let $\mathbf{g}$ denote a marker color image and $\mathbf{f}$ a mask color image (if $o_{\text {lex }}(\mathbf{g}) \leq o_{\text {lex }}(\mathbf{f})$, then $\mathbf{g} \wedge_{v} \mathbf{f}=\mathbf{g}$ ). The vectorial geodesic dilation of size 1 of the marker image $\mathbf{g}$ with respect to the mask $\mathbf{f}$ can be defined as:

$$
\delta_{V \mathbf{f}}^{(1)}(\mathbf{g})=\delta_{V}^{(1)}(\mathbf{g}) \wedge_{V} \mathbf{f}
$$

where it is very important that the infimum operation is done with the same lexicographical ordering as the vectorial dilation. This way, there are not false colors in the result.

The vectorial geodesic dilation of size $n$ of a marker color image $\mathbf{g}$ with respect to a mask color image $\mathbf{f}$ is obtained by performing $n$ successive geodesic dilations of g with respect to $\mathbf{f}$ :

$$
\delta_{v \mathbf{f}}^{(n)}(\mathbf{g})=\delta_{v \mathbf{f}}^{(1)}\left[\delta_{v_{\mathbf{f}}}^{(n-1)}(\mathbf{g})\right]
$$

with $\delta_{V \mathbf{f}}^{(0)}(\mathbf{g})=\mathbf{f}$

Geodesic transformations of bounded images always converge after a finite number of iterations [13]. The propagation of the marker image is impeded by the mask image. Morphological reconstruction of a mask image is based on this principle.

The vectorial reconstruction by dilation of a mask color image $\mathbf{f}$ from a marker color image $\mathbf{g}$, (both with the same dominion and $\left.o_{\text {lex }}(\mathbf{g}) \leq o_{\text {lex }}(\mathbf{f})\right)$ can be defined as:

$$
R_{V_{\mathbf{f}}}(\mathbf{g})=\delta_{V \mathbf{f}}^{(n)}(\mathbf{g})
$$

where $n$ is such that $\delta_{v_{\mathbf{f}}}^{(n)}(\mathbf{g})=\delta_{v_{\mathbf{f}}}^{(n+1)}(\mathbf{g})$.

The vectorial reconstruction is an algebraic opening only if all the operations between pixels respect the lexicographical order.

\section{Application: Gaussian noise removal}

Vector-connected filters will be used to eliminate Gaussian noise from color images. Chromatic Gaussian noise, in contrast to impulsive noise, it changes the entire definition of the image. Its effect is most obvious in the homogenous regions, which become spotty. With vector-connected filters we can reduce the Gaussian noise by 
merging the flat zones in the image. As such, the noisy image is simplified and the contours of the objects are preserved.

The images of our study are the color images of "Parrots" (Fig. 2.a), "Vases" (Fig. 2.c), "Lenna" (Fig. 2.e) and "Peppers" (Fig. 2.g) which has been corrupted by a Gaussian noise with a variance between 15 and 20.

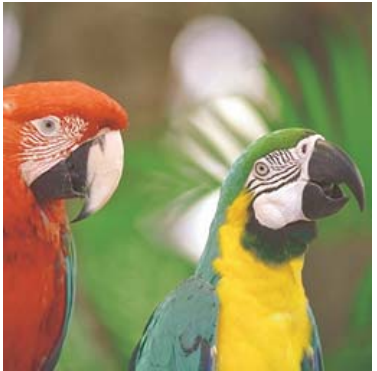

(a)

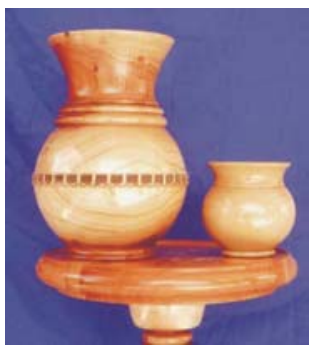

(c)

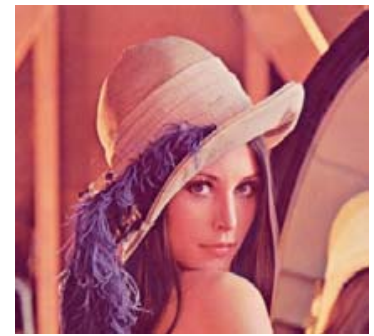

(e)

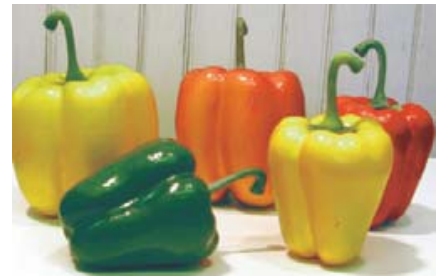

(g)

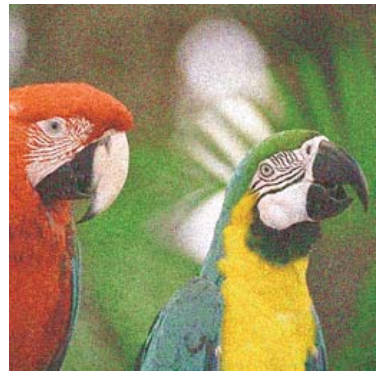

(b)

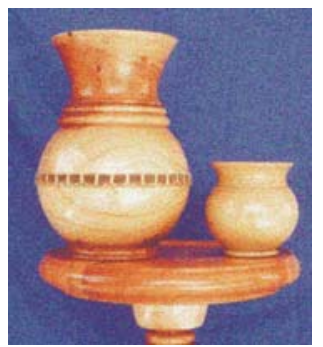

(d)

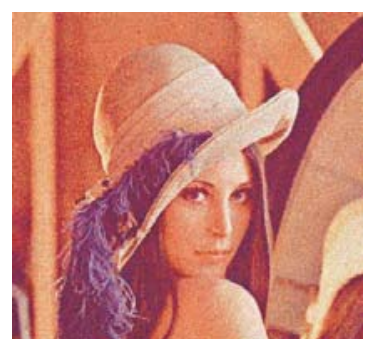

(f)

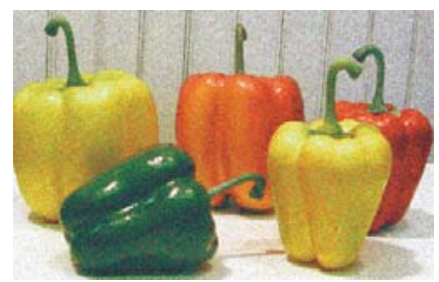

(h)

Fig. 2. Original and noisy images. (a-b) "Parrots" $\sigma=20$, (c-d) "Vases" $\sigma=15$, (e-f) "Lenna" $\sigma=18$ and (g-h) "Peppers" $\sigma=17$. 
We apply a vectorial opening by reconstruction (VOR) in the noisy image. For the morphological processing, the lexicographical ordering $o_{\text {lex }}=m \rightarrow$ saturation $\rightarrow$ hue, will be used. VOR is defined as follows:

$$
\gamma_{v_{\mathbf{f}}}^{(n)}=\delta_{V_{\mathbf{f}}}^{(n)}\left(\varepsilon_{V}{ }^{(s)}(\mathbf{f})\right)
$$

The vectorial erosion of the opening by reconstruction is made with increasing sizes (s) of the structuring element, from $5 \times 5$ to $11 \times 11$. In Figure 3, the results of the connected filter for the "Parrots" image are observed. In accordance with the increase of the structuring element, the noise is reduced, but the image is darkened (VOR is an antie-extensive operation).

We now apply a vectorial closing by reconstruction (VCR) to the original image and we obtain the results which are presented in Figure 4. In this case, the image is clarified as the size of the structuring element of the vectorial dilation is increased. This is due to the extensive operation of the VCR. VCR is defined as:

$$
\phi_{V \mathbf{f}}^{(n)}=\varepsilon_{V \mathbf{f}}^{(n)}\left(\delta_{V}^{(s)}(\mathbf{f})\right)
$$

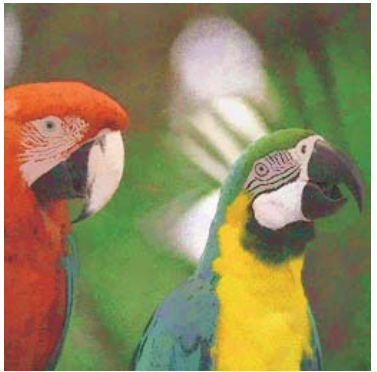

(a)

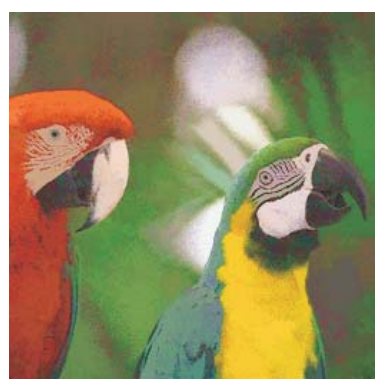

(c)

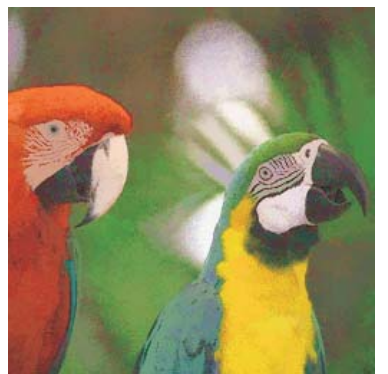

(b)

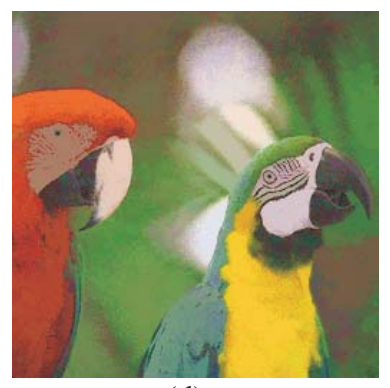

(d)

Fig. 3. Vectorial openings by reconstruction (VOR) from: 5x5-eroded image (a), 7x7-eroded image (b), 9x9-eroded image (c), 11x11-eroded image (d).

In Figure 5, the effect of reducing flat zones within the noisy image is shown. In particular, the upper left corner of the images is detailed. Figure 5.a shows the original noisy section. In Figures 5.b and 5.c, we can observe the progressive reduction of flat zones as the size of the structuring element (s) increases in the operation of erosion of the vectorial opening by reconstruction. For our purpose of 
noise removal we chose a structuring element of $7 \times 7$ as the best for preserving contours and details in the images.

In order to attenuate the dark and light effects of the previous filters, we calculate the vectorial mean of both filters. In Figure 6 we can see the art flow of the algorithm for Gaussian noise removal. The visual result of our algorithm and the final images are shown in Figure 7.

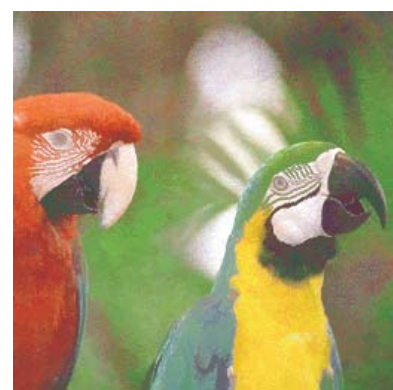

(a)

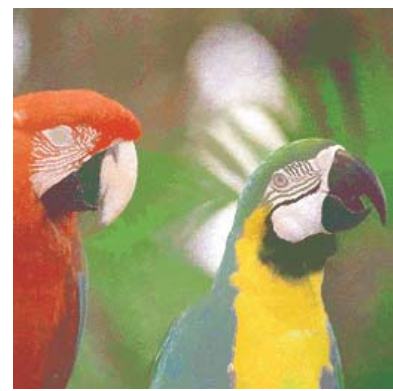

(c)

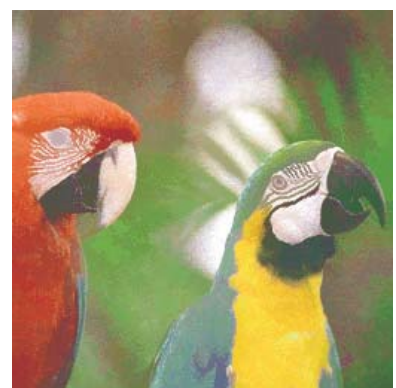

(b)

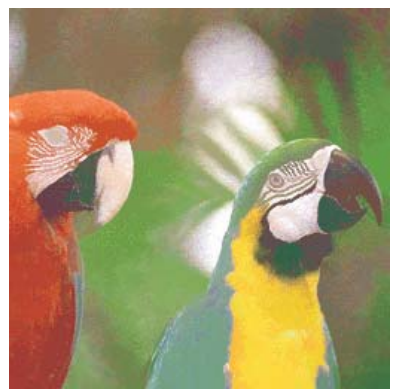

(d)

Fig. 4. Vectorial closings by reconstruction (VCR) from: 5x5-dilated image (a), 7x7-dilated image (b), 9x9-dilated image (c), 11x11-dilated image (d).

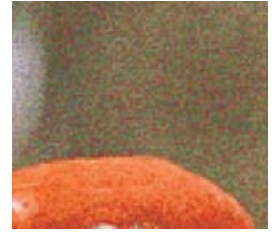

(a)

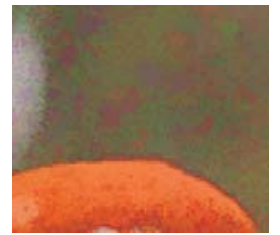

(b)

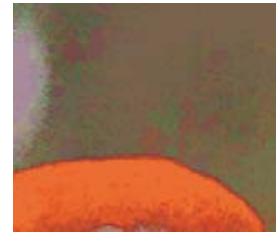

(c)

Fig. 5. Detail of simplification of the image. (a) noisy section. (b) VOR-7x7. (c) VOR$11 \times 11$.

We use the normalised mean squared error (NMSE) to assess the performance of the different means of the filters. The NMSE test for color images is calculated from the RGB tri-stimulus values [3], where $\mathbf{i}$ is the original color image and $\mathbf{f}$ is the processed image: 


$$
N M S E=\frac{\sum \sum\left[\left(i_{r}(x, y)-f_{r}(x, y)\right)^{2}+\left(i_{g}(x, y)-f_{g}(x, y)\right)^{2}+\left(i_{b}(x, y)-f_{b}(x, y)\right)^{2}\right]}{\sum \sum\left(i_{r}{ }^{2}(x, y)+i_{g}{ }^{2}(x, y)+i_{b}{ }^{2}(x, y)\right)}
$$

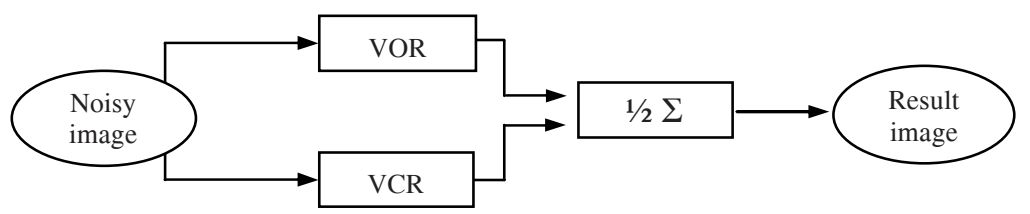

Fig. 6. The Algorithm for Gaussian noise attenuation in color images by morphological reconstruction. Structuring element (s) $7 \times 7 . o_{\mathrm{lex}}=m \rightarrow$ saturation $\rightarrow$ hue.

In addition to the NMSE test, three subjective criteria can be used:

- Visual attenuation of noise.

- Contour preservation.

- Detail preservation.

The evaluation of the quality with subjective criteria is divided into four categories: i.e., excellent (4), good (3), regular (2) and bad (1). The result of the filter on the noise-polluted images is illustrated in Table 1. The value of the NSME test for the original image and the noisy one are 0.0952 for "Parrots", 0.0892 for "Vases", 0.102 for "Lenna" and 0.919 for "Peppers".

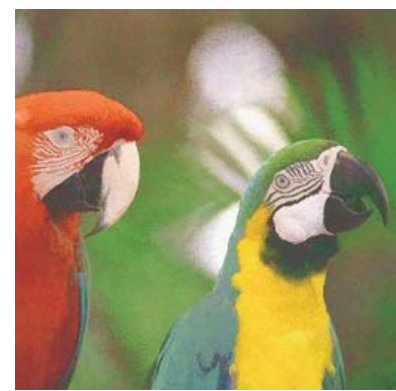

(a)

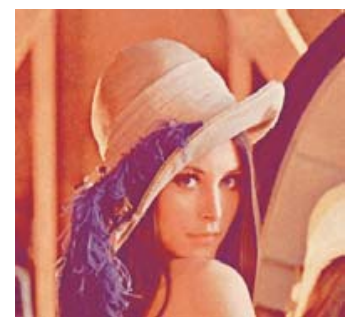

(c)

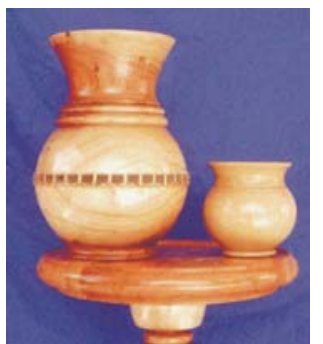

(b)

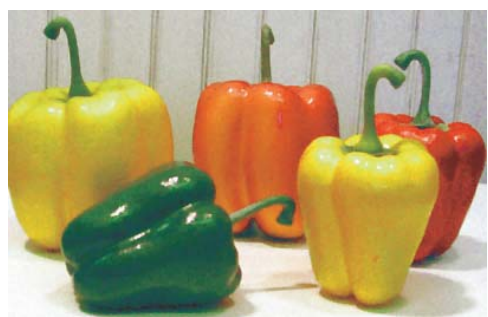

(d)

Fig. 7. Final results. Vectorial Mean of VOR-VCR in (a) "Parrots", (b) "Vases", (c) "Lenna" and (d) "Peppers". 
Table 1. Color NSME and subjective criteria. The "NSME original/noisy" is the normalised mean squared error calculated between the original image and the noisy image. The NSME "M-(VOR,VCR) " is the normalised mean squared error calculated between the original image and the filtered image.

\begin{tabular}{cccccc}
\hline & $\begin{array}{c}\text { NMSE } \\
\text { Original/ } \\
\text { noisy }\end{array}$ & $\begin{array}{c}\text { NMSE } \\
\text { Original/ } \\
\text { M-(VORVCR) }\end{array}$ & $\begin{array}{c}\text { Visual } \\
\text { attenuation of } \\
\text { noise }\end{array}$ & $\begin{array}{c}\text { Contour } \\
\text { preservation }\end{array}$ & $\begin{array}{c}\text { Detail } \\
\text { preservation }\end{array}$ \\
Parrots & 0.0952 & 0.0775 & 3 & 4 & 3 \\
Vases & 0.0892 & 0.0688 & 4 & 4 & 4 \\
Lenna & 0.102 & 0.0717 & 3 & 3 & 3 \\
Peppers & 0.0919 & 0.0597 & 4 & 4 & 4 \\
\hline
\end{tabular}

Regarding the values from the NMSE test, we should emphasize that they are all located below the value of the noisy image. The most optimal result, according to the NSME test, is the presented in the image of "Vases". Regarding the subjective evaluation of the quality of the filter, the best conservation of contours and details are already presented in the "Vases" image (noise $\sigma=15$ ). Nevertheless, visual attenuation of noise (subjective criteria) is achieved in all the images.

\section{Conclusions}

In this paper, we have presented an algorithm for eliminating Gaussian noise in color images. The geodesic method for reducing noise has been shown to be efficient for a Gaussian noise of a variance of 20. In the experiments carried out, better results where obtained for lower variances, specifically 15 .

A best elimination of noise can be obtained if the morphological elemental operations of the connected filters are made with a large structuring element, but the best conservation of structures and details in the image are achieved with a smaller structuring element. For this reason we have used a structuring element of maximum size of $7 \times 7$.

Based on the success shown by these results, we are now working on an improvement of our method for noisy removal in color images. We work in multiprocessor configurations for color geodesic operations in order to reduce the processing time these operations.

\section{References}

1. Trahanias, P., Venetsanopoulos, A.: Vector directional filters: A new class of multichannel image processing filters. IEEE Transactions on Image Processing. Vol.2, I.4 (1993) 528534.

2. Astola, J., Haavisto, P., Neuvo, Y.: Vector median filters. Proceedings of the IEEE, Vol. 78, I. 4, (1990) 678-689. 
3. Deng-Wong, P., Cheng, F., Venetsanopoulos, A.: Adaptive Morphological Filters for Color Image Enhancement. Journal of Intelligent and Robotic System, Vol. 15, (1996) 181-207.

4. Serra, J.: Image analysis and Mathematical Morphology. Vol I, and Image Analysis and Mathematical Morphology. Vol II: Theorical Advances, Academic Press, London (1982) and (1988).

5. Serra, J.: Anamorphoses and Function Lattices (Multivalued Morphology). In: E. Douguerty (Ed.): Mathematical Morphology in Image Processing, Marcel-Dekker, (1992) 483-523.

6. Hanbury, A., Serra, J.: Morphological operators on the unit circle. IEEE Transactions on Image Processing, Vol. 10, I. 12, (2001) 1842-1850.

7. Ortiz, F., Torres, F., De Juan, E., Cuenca, N.: Color Mathematical Morphology For Neural Image Análisis. Real-Time Imaging, Vol. 8 (2002) 455-465.

8. Ortiz, F.: Procesamiento morfológico de imágenes en color. Aplicación a la reconstrucción geodésica. PhD Thesis, University of Alicante (2002).

9. Ortiz, F, Torres, F., Angulo, J., Puente, S.: Comparative study of vectorial morphological operations in different color spaces. Proceedings SPIE: Algorithms, Techniques and Active Vision, vol. 4572, (2001) 259-268.

10. Peters II, A.: Mathematical morphology for angle-valued images. Proceedings of SPIE, Non-Linear Image Processing VIII , Vol. 3026, (1997) 84-94.

11. Serra, J.: Espaces couleur et traitement d'images. Tech. Report N-34/02/MM. Centre de Morphologie Mathématique, École des Mines de Paris (2002).

12. Ortiz, F., Torres, F.: Real-time elimination of specular reflectance in colour images by 2D-histogram and mathematical morphology. Journal of Imaging Science and Technology. Special issue on Real-Time Imaging. Vol. $49 \quad$ (2005) 220- 229.

13. Ortiz, F., Torres, F.: Vectorial Morphological Reconstruction for Brightness Elimination in Colour Images. Real Time Imaging. Vol. 10 (2004) 379-387.

14. Salembier, P., Serra, J.: Flat zones filtering, connected operators, and filters by reconstruction. IEEE Transactions on Image Processing, Vol.4, I.8, (1995) 1153-1160.

15. Soille, P. Morphological Image Analysis. Principles and Applications. Springer-Verlag, Berlin (1999). 